\title{
AVALIAÇÃO DO EQUILÍBRIO DE MULHERES COM CÂNCER DE MAMA
}

\author{
Valeria Bruna de Santana Moura ${ }^{1}$, Doralice Daieli Mendonça Farias Tavares ${ }^{1}$, Beatriz Jeronymo Schwartz ${ }^{1}$, \\ Sara Llorente Cordeiro ${ }^{1}$, Aline Duarte Ferreira ${ }^{1}$, Bruna Corral Garcia de Araujo ${ }^{1}$, Ismael Forte Freitas Junior ${ }^{2}$, \\ Flavia de Souza Lima Matias ${ }^{1}$, Tainá Oliveira Lopes ${ }^{1}$, Tainara Fernanda da Silva Santos ${ }^{1}$, Ana Claudia de \\ Souza Fortaleza ${ }^{1}$. \\ ${ }^{1}$ Universidade do Oeste Paulista - UNOESTE, Presidente Prudente, SP; ${ }^{2}$ Universidade Estadual Paulista - UNESP, \\ Presidente Prudente, SP. e-mail: valeria.santana.moura@hotmail.com
}

\section{RESUMO}

O objetivo desse estudo foi verificar o equilíbrio de mulheres com câncer de mama. $O$ estudo foi transversal e avaliou o equilíbrio de 15 mulheres, sendo oito mulheres com câncer e sete sem câncer. A avaliação foi realizada por meio do Mini Balance Evaluation Systems (MiniBESTest), TUG simples e dupla tarefa. A média de idade foi de 59,2 \pm 5 anos para o grupo de mulheres com câncer e $60,2 \pm 5$ anos para o grupo controle. $\mathrm{O}$ Teste $\mathrm{t}$ Student para amostras independentes mostrou que não houve diferença estatística entre os grupos para as variáveis: pontuação no MiniBESTest, tempo de realização do TUG simples e tempo de realização do TUG dupla tarefa. Mulheres com câncer não apresentaram alterações de equilíbrio comparadas ao grupo controle de acordo com o MiniBESTest, e TUG tarefa simples e dupla tarefa.

Palavras-chave: Terapêutica, equilíbrio postural, neoplasias da mama, mastectomia, quimioterapia.

\section{EVALUATION OF BALANCE OF WOMEN WITH BREAST CÂNCER}

\section{ABSTRACT}

The objective of this study was to verify the balance of women with breast cancer. This cross-sectional study, tested balance of 15 women, eight women with cancer and seven without cancer. The evaluation was performed through the Mini Balance Evaluation Systems (MiniBESTest), TUG with simple and dualtask. The mean of age was $59.2 \pm 5$ years for cancer group and $60.2 \pm 5$ years for the control group. Student's t-test for independent samples showed that there was no statistical difference between the groups for: MiniBESTest score, TUG with simple and dual-task. Women with cancer did not present balance impairments compared to control group according to MiniBESTest, and TUG with simple and dual-task.

Keywords: Therapeutics, postural balance, breast neoplasms, mastectomy, drug therapy.

\section{INTRODUÇÃO}

O câncer de mama é o tipo de câncer mais incidente e que possui a maior taxa de mortalidade entre as mulheres no mundo ${ }^{1}$. No Brasil essa taxa é altamente significativa, estimando-se 59,700 novos casos para cada ano biênico 2018-2019, sendo esperado um risco de $56,33 \%$ a cada 100 mil mulheres ${ }^{2}$. Várias estratégias são desenvolvidas para detecção precoce do câncer de mama, uma vez que a chance de tratamento e cura é maior quando detectado em fases iniciais ${ }^{3}$.

O câncer de mama possui diferentes abordagens de tratamento, dentre eles o cirúrgico (técnicas conservadoras e radicais) e o tratamento adjuvante (quimioterapia, radioterapia e hormonioterapia). Além disso, tratamentos com terapia hormonal são 
empregados afim de minimizar o surgimento de tumores secundários ${ }^{4}$.

As mastectomias são divididas em mastectomias conservadoras e radical. $\mathrm{Na}$ mastectomia conservadora o procedimento é cirúrgico com a finalidade da retirada total da mama sem exceção do mamilo. Quando realizado o procedimento em ambas as mamas recebe o nome de mastectomia bilateral. Esse tipo de mastectomia é indicado para a prevenção em mulheres com grandes possibilidades de adquirir o câncer de mama. A mastectomia radical também é um procedimento cirúrgico, no qual a mama toda é removida, porém incluindo os músculos peitorais da parede torácica e os linfonodos axilares ${ }^{5,6}$.

Em pacientes com mastectomias unilaterais, podem ser observadas assimetrias posturais ${ }^{7}$, essas assimetrias causam desarranjos posturais que consequentemente provocam alterações do equilíbrio que por sua vez resulta em alterações da marcha ${ }^{8}$.

A quimioterapia atinge tanto células neoplásicas quanto células saudáveis causando diversos efeitos colaterais como náusea e vômito, fadiga, alopecia, neurogenia, diarreia, constipação intestinal, alterações do sistema tegumentar e as neurotoxicidades ${ }^{9}$. Uma das formas de apresentação da neurotoxicidade é a neuropatia periférica ${ }^{10}$. As neuropatias provocam déficit de força muscular e diminuição da sensibilidade tátil e proprioceptiva nas mãos e pés ${ }^{11}$. Além disso, todas essas alterações podem levar ao aumento da morbidade, restrição nas atividades de vida diária, quedas e fraturas ${ }^{12}$.

Para avaliação do equilíbrio vem sendo utilizado o Mini Balance Evaluation Systems (MiniBESTest), uma versão reduzida do Balance Evaluation Systems (BESTest), de mais fácil aplicabilidade. $O$ MiniBESTest avalia componentes importantes do equilíbrio como respostas posturais reativas, orientação sensorial, ajustes posturais antecipatórios, e estabilidade na marcha. Apresenta altos níveis de confiabilidade $^{13}$, e precisão dos resultados em mulheres com câncer de mama ${ }^{14}$. Portanto detectar alterações de equilíbrio precocemente se faz necessário para implantar estratégias de tratamentos adequados.

O objetivo desse estudo foi verificar o equilíbrio de mulheres com câncer de mama.

\section{METODOLOGIA}

Este estudo seguiu os princípios da declaração de Helsinque revisada em 2008 e foi aprovado pelo Comitê de Ética (CAAE 90901318.9.0000.5515), atendendo às normas da resolução 466/2012. Os participantes inseridos no estudo foram instruídos em relação a todos os procedimentos que foram executados e assinaram o termo de consentimento livre e esclarecido concordando em participarem do estudo.

Trata-se de um estudo transversal, que fez parte de um projeto maior de pesquisa sobre os efeitos do treinamento aeróbio em mulheres em tratamento para câncer de mama: ensaio clínico randomizado. As avaliações foram realizadas no Centro de Estudos e Laboratório de Avaliação e Prescrição de Atividade Motora (CELAPAM), no departamento de Educação Física da Universidade Estadual Paulista - UNESP, em Presidente Prudente SP, Brasil, no mês de dezembro de 2018 a janeiro de 2019.

O número de indivíduos avaliados foi de 15 pacientes, sendo divididos em dois grupos: Grupo de mulheres com câncer de mama (GCM, $\mathrm{n}=8$ ) e grupo de mulheres sem câncer de mama (GSCM, n=7), sendo essa população a mesma recrutada no projeto maior, tanto grupo experimental quanto grupo controle. Foram incluídas no estudo mulheres entre 55 a 65 anos, que apresentaram diagnóstico de câncer de mama nos estágios 1 a 3 da doença, que possuíram liberação médica para realizar as avaliações, que não possuíam alterações ortopédicas e musculares ou limitações físicas que impeça a realização das avaliações estando assim hemodinamicamente estáveis. Foram excluídas pacientes que apresentaram descompensações hemodinâmicas.

O equilíbrio foi avaliado por meio do MiniBESTest ${ }^{12}$. O teste, já é confiável e validado no Brasil $^{15}$, possuindo 14 itens com duração de aplicação de 10 a $15 \mathrm{~min}$, o que torna mais fácil e rápido a execução. Ele é capaz de rastrear déficits de controle postural estático e dinâmico em vários tipos de situações nas atividades diárias. A pontuação máxima é de 28 e a pontuação mínima é de 0 pontos, cada item sendo pontuado de 0 a 2, na qual 2 significa normal, 1 moderado e 0 grave, ou seja, quanto maior a pontuação do indivíduo melhor o equilíbrio. Este teste já foi previamente testado em pessoas idosas sobreviventes de câncer e mostrou alta confiabilidade e validade para esta população ${ }^{14}$. 
O teste Timed Up \& Go (TUG) é o item 14 da avaliação do MiniBESTest. Devido a sua importância funcional ${ }^{16}$ este teste (com tarefa simples e dupla tarefa) foi analisado separadamente.

As variáveis foram verificadas quanto à normalidade com o teste de Shapiro-Wilk. Os dados paramétricos foram expressos em média e desvio-padrão. A comparação entre cada grupo foi realizada através do teste $t$ Student para amostras independentes. Todas as análises foram realizadas no software SPSS versão 24.0 e a significância estatística de $5 \%$ foi adotada.

\section{RESULTADOS}

Participaram do estudo 15 mulheres, com média de idade de $59,2 \pm 5$ anos no GCM e $60,2 \pm 5$ anos no GSCM, não existindo diferença significante entre os grupos para esta variável.

Nas Tabelas 1 e 2 são mostrados dados descritivos do GCM e GSCM, respectivamente, relacionados à porcentagem de mulheres que apresentou resposta normal, moderada e grave em cada uma das atividades do MiniBESTest.

Os 14 componentes do MiniBESTest e seus resultados que podem ser considerados normal, moderado e grave estão apresentados para os grupos com e sem câncer nas Tabelas $1 \mathrm{e}$ 2 , respectivamente. No teste ficar na ponta dos pés o grupo com câncer de mama apresentou pior resultado, pois uma porcentagem de mulheres obteve classificação grave, já nos testes de correção com passos compensatórios os dois grupos apresentaram dificuldade não apresentando a classificação normal em nenhum desses itens.

Quando realizado o Teste $\mathrm{t}$ Student para amostras independentes na comparação entre os grupos, não houve diferença estatística $(p>0,05)$ para as variáveis: pontuação no MiniBESTest, tempo de realização do TUG simples, tempo de realização do TUG dupla tarefa para o GCM e GSCM (Tabela 3).

Tabela 1. Grupo de mulheres com câncer de mama (GCM) avaliadas segundo MiniBESTest.

\begin{tabular}{lccc}
\hline Testes & Normal (\%) & Moderado (\%) & Grave (\%) \\
\hline Sentado para de pé & 100 & 0 & 0 \\
Ficar na ponta dos pés & 87,5 & 0 & 12,5 \\
De pé em uma perna (direita) & 87,5 & 12,5 & 0 \\
De pé em uma perna (esquerda) & 50 & 50 & 0 \\
Correção com passo compensatório para frente & 62,5 & 37,5 & 0 \\
Correção com passo compensatório para trás & 25 & 75 & 0 \\
Correção com passo compensatório para lateral (direita) & 62,5 & 37,5 & 0 \\
Correção com passo compensatório para lateral (esquerda) & 62,5 & 37,5 & 0 \\
Olhos abertos superfície firme pés juntos & 100 & 0 & 0 \\
Olhos fechados superfície de espuma pés juntos & 100 & 0 & 0 \\
Inclinação olhos fechados & 100 & 0 & 0 \\
Mudança na velocidade de marcha & 100 & 0 & 0 \\
Andar com viradas de cabeça horizontal & 62,5 & 37,5 & 0 \\
Andar e girar sobre o eixo & 87,5 & 12,5 & 0 \\
Passar sobre obstáculos & 62,5 & 37,5 & 0 \\
TUG e TUG dupla tarefa & 50 & 25 & 25 \\
\hline \hline
\end{tabular}

TUG: TimedUp \& Go. 
Tabela 2. Grupo de mulheres sem câncer de mama (GSCM) avaliadas segundo MiniBESTest.

\begin{tabular}{lccc}
\hline Testes & Normal (\%) & Moderado (\%) & Grave (\%) \\
\hline Sentado para de pé & 100 & 0 & 0 \\
Ficar na ponta dos pés & 100 & 0 & 0 \\
De pé em uma perna (direita) & 100 & 0 & 0 \\
De pé em uma perna (esquerda) & 85,7 & 14,3 & 0 \\
Correção com passo compensatório para frente & 71,5 & 28,5 & 0 \\
Correção com passo compensatório para trás & 42,9 & 42,8 & 14,3 \\
Correção com passo compensatório para lateral (direita) & 42,9 & 42,8 & 14,3 \\
Correção com passo compensatório para lateral (esquerda) & 42,9 & 42,8 & 14,3 \\
Olhos abertos superfície firme pés juntos & 100 & 0 & 0 \\
Olhos fechados superfície de espuma pés juntos & 100 & 0 & 0 \\
Inclinação olhos fechados & 100 & 0 & 0 \\
Mudança na velocidade de marcha & 100 & 0 & 0 \\
Andar com viradas de cabeça horizontal & 85,7 & 14,3 & 0 \\
Andar e girar sobre o eixo & 56,2 & 43,8 & 0 \\
Passar sobre obstáculos & 85,7 & 14,3 & 0 \\
TUG e TUG dupla tarefa & 73,4 & 14,3 & 14,3 \\
\hline \hline
\end{tabular}

TUG: TimedUp \& Go.

Tabela 3. Pontuação MiniBESTest, tempo do TUG, TUG dupla tarefa e idade para o GCM e GSCM.

\begin{tabular}{cccc}
\hline & GCM & GSCM & \\
& Média \pm DP & Média \pm DP & P \\
\hline Pontos MiniBESTest & $24,1 \pm 2,7$ & $24,8 \pm 2,2$ & 0,304 \\
TUG & $7,7 \pm 1,4$ & $7,0 \pm 0,8$ & 0,83 \\
TUG dupla tarefa & $8,4 \pm 1,0$ & $7,9 \pm 1,3$ & 0,147 \\
Idade & $59,2 \pm 2,4$ & $60,2 \pm 3,9$ & 0,424 \\
\hline \hline
\end{tabular}

\section{DISCUSSÃO}

Foi possível verificar que não houve diferença significativa entre os grupos para a pontuação no MiniBESTest. Este estudo também verificou separadamente o tempo de realização do TUG com e sem dupla tarefa, nos quais também não foram encontrados significância.

O TUG tem sido amplamente utilizado na área clínica devido a sua fácil aplicabilidade, pois é capaz de detectar o declínio da mobilidade física com o decorrer da idade ${ }^{17}$ podendo ocasionar quedas. Mulheres com câncer de mama apresentaram maior dificuldade nas tarefas de equilíbrio estático e diminuição na velocidade da marcha a curtas e longas distâncias quando comparadas a mulheres sem câncer de mama $^{17}$.
Com relação ao MiniBESTest, possui alto nível de confiabilidade $^{13}$ e validade para sobreviventes de câncer de mama, por essa razão esse instrumento foi utilizado. 0 resultado desse teste pode ser usado como direcionamento para o tratamento de deficiências específicas de equilíbrio em sobreviventes de câncer ${ }^{14}$.

A quimioterapia tem como um dos efeitos colaterais a neuropatia periférica, na qual, a mesma provoca déficit de força muscular e equilíbrio, sendo comprovado que a atividade física demonstrou melhora significativa nessas funções $^{18}$.

A postura corporal equilibrada é mantida por uma função complexa dependente da interação do sistema neural, por meio de estruturas proprioceptivas e exteroceptivas do sistema musculoesquelético. Mulheres com 
câncer de mama adotam posturas antálgicas compensatórias após o tratamento cirúrgico, a fim de esconder a falta da mama e evitar dores. A mulher altera a sua biomecânica postural na tentativa de se reequilibrar, ocasionando com frequência contraturas na região cervical e cintura escapular, abduzindo escápula homolateral à cirurgia e elevando o ombro e escápula ${ }^{19,20}$. Mulheres com câncer de mama que realizaram tratamentos cirúrgicos, após a realização de uma avaliação postural através da fotogrametria computadorizada, foram detectadas alterações posturais sendo relacionados principalmente à protrusão de ombro homolateral à intervenção cirúrgica e a anteriorização ${ }^{21}$.

Apesar de acreditarmos que poderia haver uma diferença entre os grupos nos testes utilizados porque mulheres com câncer de mama podem apresentar diversas alterações como déficits sensoriais e de força muscular decorrentes da neuropatia periférica, quando em tratamento com quimioterapia ${ }^{18}$, e alterações posturais, no caso de tratamentos cirúrgicos ${ }^{21}$, que estão diretamente relacionadas ao equílibrio $^{22}$, isso não foi comprovado provavelmente devido ao pequeno número de indivíduos, e ao fato de termos uma amostra heterogênea, com mulheres com câncer de mama sendo algumas mastectomizadas ou que realizaram tratamento com quimioterapia e outras que realizaram outros tratamentos.

Acredita-se que detectar alterações no equilíbrio precocemente se faz necessário para implantar estratégias de tratamento conservador, já que o equilíbrio normal é indispensável para a estabilidade e mobilidade durante as atividades funcionais ${ }^{23}$.

Sugere-se a realização de um novo estudo, com maior número amostral, sendo que as mulheres com câncer de mama deverão ter em comum a mastectomia e realizado tratamento com quimioterapia, sendo necessária também a avaliação da neuropatia periférica. $O$ desenvolvimento de novas pesquisas, que visem desenvolver estratégias de prevenção, redução de ocorrências e tratamento da neuropatia periférica é de extrema importância ${ }^{24}$.

Assim este estudo contribui com o âmbito da pesquisa por alertar pesquisadores sobre a importância de avaliar o equilíbrio em mulheres com câncer de mama, doença que apresenta um grande número de pessoas afetadas, para que estes possam realizar pesquisas com maior número de mulheres e comprovar nossa hipótese inicial. Além disso, apresentamos neste estudo uma avaliação nunca antes utilizada no Brasil nesta população e que pode ser facilmente aplicada na prática clínica e pesquisa sendo de alta confiabilidade ${ }^{14}$.

Mulheres com câncer de mama não apresentaram alterações de equilíbrio comparadas ao grupo controle de acordo com o MiniBESTest e TUG tarefa simples e dupla tarefa.

\section{AGRADECIMENTOS E CONFLITO DE INTERESSE}

Agradecemos a contribuição da equipe do Centro de Estudos e Laboratório de Avaliação e Prescrição de Atividade Motora (CELAPAM), por cederem o espaço e os instrumentos utilizados para a avaliação do equilíbrio de mulheres com câncer de mama.

Agradecemos a UNOESTE pelo suporte em estrutura para realização deste trabalho.

Os autores declaram não haver conflitos de interesse.

\section{REFERÊNCIAS}

1. Chaker L, Falla A, Lee SJVD, Muka T, Imo D, Jaspers $L$ et al. The global impact of noncommunicable diseases on macro- economic productivity: a systematic review. Eur J Epidemilol. 2015;30(5):357-95. DOI: https://doi.org/10.1007/s10654-015-0026-5

2. Brasil. Ministério da saúde. INCA [internet]. Instituto Nacional de Câncer José Alencar Gomes da Silva. Estimativa 2018: incidência de câncer no Brasil. Rio de Janeiro (RJ): Ministério da saúde; 2017. [citado em 2019]. Disponível em: https://www.inca.gov.br/sites/ufu.sti.inca.local/fi les//media/document//estimativa-incidencia-decancer-no-brasil-2018.pdf

3. Migowski A, Silva GA, Dias MBK, Diz MDPE, Sant'Ana DR, Nadanovsky P. Diretrizes para deteç̧ão precoce do câncer de mama no Brasil. II - Novas recomendações nacionais, principais evidências e controvérsias. Cad Saúde Pública. 2018;34(6):e00074817.

https://doi.org/10.1590/0102-311×00074817

4. Guedes JBR, Guerra MR, Alvim MM, Leite ICG. Factors associated with adherence and persistence to hormonal therapy in women with breast cancer. Rev Bras epidemiol. 2017;20(4):636-49. DOI: 
http://dx.doi.org/10.1590/1980-

\section{$\underline{5497201700040007}$}

5. Giuliano AE, Hunt KK, Ballman KV, Beitsch PD, Whitworth PW, Blumencranz PW et al. Axillary dissection vs no axillary dissection in women with invasive breast cancer and sentinel node metastasis: a randomized clinical trial. Jama. 2011;305(6):569-75.

DOI: https://doi.org/10.1001/jama.2011.90

6. Silva SED, Vasconcelos EV, Santana ME, Rodrigues ILA, Leite TV, Santos LMS, et al. Representações sociais de mulheres mastectomizadas e suas implicações para o autocuidado mastectomizadas e suas implicações para o autocuidado. Rev Bras Enferm. 2010; 63(5):727-34.

DOI:

http://dx.doi.org/10.1590/S0034-

$\underline{71672010000500006}$

7. Rostkowska E, Bak M, Samborski W. Body posture in women after mastectomy and its changes as a result of rehabilitation. Adv Med Sci. 2006;51:287-97.

8. Dye DC, Eakman AM, Bolton KM. Assessing the Validity of the Dynamic Gait Index in a Balance Disorders Clinic: An Application of Rasch Analysis. Phy Ther. 2013;93(6):809-18. DOI: https://doi.org/10.2522/ptj.20120163

9. Bonassa EMA, Santana TR. Enfermagem em terapêutica oncológica. 3.ed. Rio de Janeiro: Atheneu; 2005.

10. Afonseca SO, Silva MAC, Giglio AD. Abordagem da neuropatia periférica induzida por quimioterapia. Rev Bras Med. 2010;67(n.esp):205.

11. Schimitt AC, Repka CP, Heise GD, Challis JH, Smith JD. Comparison of posture and balance in cancer survivors and age-matched controls. Clin Biomech. 2017;50:1-6. DOI: https://doi.org/10.1016/j.clinbiomech.2017.09.0 $\underline{10}$
12. Horak FB, Wrisley DM, Frank J. The Balance Evaluation Systems Test (BESTest) to differentiate balance deficits. Phy Ther. 2009;89(5):484-98. DOI: https://doi.org/10.2522/ptj.20080071

13. Godi M, Franchignoni F, Caligari H, Giordano Um, Turcato AM, Nardone A. Comparison of Reliability, Validity, and Responsiveness of the Mini-BESTest and Berg Balance Scale in Patients With Balance Disorders. Phy Ther. 2013;93(2):158-67. DOI: https://doi.org/10.2522 $\angle$ ptj.20120171

14. Huang $\mathrm{MH}$, Miller $\mathrm{K}$, Smith $\mathrm{K}$, Fredrickson $\mathrm{K}$, Shilling T. Reliability, validity, and minimal detectable change of balance evaluation systems test and its short versions in older cancer survivors: a pilot study. J Geriatr Phys Ther. 2016;39(2):58-63.

DOI: https://doi.org/10.1519/JPT.0000000000000047

15. Bambirra C, Magalhães LC, Paula FR. Confiabilidade e validade do BESTest e do MiniBESTest em hemiparéticos crônicos. Rev Neurocienc. $\quad 2015 ; 23(1): 30-40 . \quad$ DOI: https://doi.org/10.4181/RNC.2015.23.01.943.11p

16. Vieira WO, Ostolin TLVP, Ferreira M, Sperandio $E F$, Dourado VZ. Test timed up and go and its correlation with age and functional exercise capacity in asymptomatic women. Fisioter Mov. 2017; 30(3):463-71. DOI: http://dx.doi.org/10.1590/1980-

5918.030.003.A004

17. Hsieh KL, Wood TA, An R, Trinh L, Sosnoff JJ. Gait and Balance Impairments in Breast Cancer Survivors: A Systematic Review and Meta-analysis of Observational Studies. Arch Rehabil Res Clin Transl. 2019;1(1):1-14. DOI: https://doi.org/10.1016/i.arrct.2018.12.001

18. Zimmer $P$, Trebing $S$, Timmers-Trebing $U$, Schenk A, Paust R, Bloch $W$ et al. Eight-week, multimodal exercise counteracts a progress of chemotherapy-induced peripheral neuropathy and improves balance and strength in metastasized colorectal cancer patients: a randomized controlled trial. Support Care Cancer. 
19. Rostkowska E, Bak M, Samborski W. Body posture in women after mastectomy and its changes as a result of rehabilitation. Adv Med Sci. 2006;51:287-97.

20. Ciesla S, Polom K. The effect immediate breast reconstruction with Becker-25 prosthesis on the preservation of proper body posture in patients after mastectomy. Eur J Surg Oncol. 2010;36(7):625-31. DOI: https://doi.org/10.1016/j.ejso.2010.05.005

21. Melo MSI, Maia JN, Silva DAL, Carvalho CC. Avaliação Postural em Pacientes Submetidas à Mastectomia Radical Modificada por meio da Fotogrametria Computadorizada. Rev Bras Cancer. 2011;57(1):39-48.

22. Macedo BG, Pereira LSM, Gomes PF, Silva JP, Castro ANV. Impacto das alterações visuais nas quedas, desempenho funcional, controle postural e no equilíbrio dos idosos: uma revisão de literatura. Rev Bras Geriatr Gerontol. 2008;11(3):419-32. DOI: http://dx.doi.org/10.1590/1809$\underline{9823.2008 .110310}$

23. Noohu MM, Dey AB, Hussain ME. Relevance of balance measurement tools and balance training for fall prevention in older adults. Journal of Clinical Gerontology \& Geriatrics. 2014;(5):315.

DOI: https://doi.org/10.1016/j.jcgg.2013.05.002

24. Costa TC, Lopes M, Anjos ACY, Zago MMF. apy-induced peripheral neuropathies: an integrative review of the literature. Rev Esc Enferm USP. 2015;49(2):332-41. DOI: https://doi.org/10.1590/S0080$\underline{623420150000200020}$ 